\title{
Erratum to: 44th Annual Scientific Meeting of the European Society for Paediatric Nephrology
}

\author{
Under the Auspices of the President of the Republic of Croatia, Ivo Josipović 14 - 17 \\ September 2011, Hotel Croatia, Cavtat - Dubrovnik, Croatia
}

Published online: 8 October 2011

(C) IPNA 2011

\section{Erratum to: Pediatric Nephrology DOI 10.1007/s00467-011-1955-1}

We sincerely apologize to the authors whose abstract was published incorrectly

\author{
S. $\operatorname{Mir}^{1}$, N. Dincel ${ }^{* 1}$, O.D. $\operatorname{Kar}^{1}$, A. Keskinoğlu ${ }^{1}$ \\ ${ }^{1}$ Ege University Medical Faculty, Izmir, Turkey
}

\section{Is the effectiveness of continue venovenous hemodiafiltration related to rifle criteria and etiology of renal failure?}

Renal replacement therapy has evolved from the need to treat the dysfunction of a single organ.As intensive care units have become more and more complex, it has become clear that the majority of patients with acute renal failure (ARF) often have dysfunction of several other organs. In order to facilitate multi organ support, continue renal replacement therapy (CRRT) techniques have been developed. The proper goal in intensive care units (ICU) is, multiorgan support therapy.For this supporting therapy, continue venovenous hemodiafiltration (CVVHDF) is becomed commonly used.We aimed to mark the relationship between CVVHDF success and etiology of renal disease. Patients having CVVHDF in different ICUs during the last 2 year period, consulted with us were included to study. Patients with acute renal failure treated with other dialysis modalities were excluded to study. Clinical data of patients were collected from the charts of patients retrospectively. Their mean age, primary cause, time of HDF need, concultation time to us and duration time of HDF were noted.

The online version of the original article can be found at http://dx.doi. org/10.1007/s00467-011-1955-1.
Patients were classified into two groups according to their primary etiology of disease, as cardiac and non-cardiac group. Cause and severity of renal failure, were assesed at the time of HDF initiation. Stage of renal failure was evaluated according to RIFLE criteria. Outcome is categorized as primary and secondary. Primary outcome was accepted as the composite correction of uremia, acidosis and methabolic parameters, and regression of fluid overload. Secondary outcomes, were improvement of hemodynamic instability and survival. Clinical severity is classified according to the APACHE score. Also, cardiac exams, ventilatory need,stay lenght in ICU and cause of deaths were noted down.

Totally charts of 36 patients,with a mean age of 6.9 years, were scanned. The mean concultation time was 38.7 hours of ICU stay. There were 9 patients and 27 patients in group 1 and 2 respectively. The majority of diagnosis were total abnormality of major vessels, ventricular septal defects and tetralogy of fallot in group1. Whereas there were heterojenity of etiologies in gruop 2, such as; 2 cases of tumor lysis syndrome, 3 with methabolic diseases, 3 traumas, 4 multiorgan disease accompanied with sepsis, 1 meningococsemia, 1 acute renal graft rejection, 1 SLE activation. 6/ 9 patients of group 1 were in RIFLE class failure and 13/27 were in risk, $8 / 27$ were in injury and the last 6 were in the failure class in group 2 when they consulted with us. The mean initiation time of CVVHDF were 2,8 hours after they had been consulted to us. They received 80.6 hours and 35 hours of dialysis, in group 1 and 2 respectively. As their responce is better in group1, dialysis time was shorter than the second group. Although no statistically significant differences were seen in primary outcome $(88,8 \%$ in group 1 and $85.1 \%$ in group $2 ; \mathrm{p}>0.05$ ) between both groups, secondary outcome were less in group 2 ameliorately. This was due to the mortality rates which were $77,7 \%$ in group 1 and $51,8 \%$ in group $2(\mathrm{p}<0.01)$. High APACHE score, decreased cardiac 
ejection fraction, arthmias, longer need for ventilatory support, sepsis were frequent in cardiac patients even if they had not proceeded to acute renal failure or multi organ disease.Our results showed that, CVVHDF treatment is successful even if in cardiac patients having with high mortality rate and been in the later stage of acute renal failure. We think that whenever these highly mortal cases were consulted in earlier stages of acute renal failure, secondary outcome of CVVHDF will be more succesfull as well as its primary outcome.

Torun Bayram, M.*1, Alaygut, D. ${ }^{1}$, Soylu, A. ${ }^{1}$, Cakmakci, H. ${ }^{1}$, Turkmen, M. A. ${ }^{1}$, Kavukcu, $\mathrm{S}^{1}$.

${ }^{1}$ Dokuz Eylul University Medical Faculty Department of Pediatrics, Izmir, Turkey

\section{Clinical and radiological course of simple renal cysts in children}

AIM: We aimed to evaluate the clinical and radiological course of simple renal cysts that are rarely seen in children.

Patients and Methods: Hospital files of the children with simple renal cysts were evaluated retrospectively for age at diagnosis, presenting complaint, follow-up period, complications and change in the cyst diameter during follow-up. RESULTS: Simple renal cyst was detected in 9 (6 male) patients. Mean age at diagnosis was $73 \pm 42$ (14-144) months and mean follow-up period of the 6 patients with

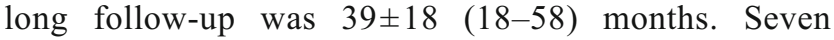
patients were diagnosed incidentally during ultrasonographic evaluation for other medical problems (obesity 2, urinary tract infection 2, voiding symptom 2 and glomerulonephritis 1). Two patients with large cysts had symptoms related to cyst (abdominal pain and abdominal mass +hematuria). Most $(n=7)$ of the cysts were in right kidney, usually in the lower pole $(n=4)$. Mean cyst diameter was $25.0 \pm 29.7$ and $37.4 \pm 59.1 \mathrm{~mm}$ at diagnosis and at last visit, respectively. Cyst size increased during follow-up in four patients $(37.3 \pm 42.1$ vs $58.5 \pm 74.6 \mathrm{~mm})$. One patient was undergone nephrectomy due to rapid increase in cyst diameter $(170 \mathrm{~mm})$, renal artery/vein compression and massive hematuria.

CONCLUSION: Simple renal cysts are rarely seen in children and usually diagnosed incidentally during ultrasonographic evaluation for other reasons. Regular radiological follow-up is important as these cysts may increase in size and become complicated.
A.G. Mitsioni*1 ${ }^{1}$, E. Siomou ${ }^{2}$, I. Bouba ${ }^{1}$, S. Petridi ${ }^{1}$, A. Siamopoulou $^{2}$, I. Georgiou ${ }^{1}$

${ }^{1}$ Laboratory of Human Reproductive Genetics, Medical School, University of Ioannina, ${ }^{2}$ Department of Pediatrics, University Hospital of Ioannina

\section{Report on polymorphism of robo2 gene in children with isolated vur and vur accompanied by renal hypodysplasia}

OBJECTIVES AND STUDY: Vesico-ureteric reflux (VUR) and VUR accompanied by renal hypodysplasia (VURRHD) may cause reflux nephropathy, resulting in chronic kidney disease in infants and young children. The aim of the present study was to evaluate the presence of sequence variations in the $\mathrm{ROBO} 2$ gene in children with familial VUR, sporadic VUR and VUR-RHD.

METHODS: A total of 103 patients were screened by single strand conformation polymorphism (SSCP) electrophoresis, in two different running conditions. Subsequently the samples with variations were characterized by direct DNA sequencing. We also screened 200 controls agematched with patients, using a restriction enzyme. The chisquare test was carried out for the statistical analysis. Also in silico analysis was performed using online available ESEfinder and SplicePort to evaluate the polymorphism's interference in splice sites.

RESULTS: The Single Nucleotide Polymorphism (SNP) rs $9874095(\mathrm{G}>\mathrm{A}$ ) was found in intron 1, close to exon 2 in the patient group. This SNP (heterozygotes, GA) was detected in $26.2 \%$ of the patients. No homozygotes in the polymorphism were found in the patient group. Among controls, 32\% were heterozygotes and $3.5 \%$ homozygotes to the polymorphism (AA). The remaining $73.8 \%$ of patients and $64.5 \%$ of controls were homozygous for the ancestral allele (GG). Both populations, patients and controls, are in Hardy-Weinberg disequilibrium. The chisquare test demonstrated a statistically significant difference between allele frequencies but not in the genotypes. In silico analysis showed a low possibility for the corresponding splice site to be affected.

CONCLUSIONS: The results so far indicate that mutations in ROBO2 are not a major cause of the anomalies in our studied patients, even though certain mutations have been found before in such patients. However VUR and VURRHD are multifactorial disorders and even a single polymorphism in association with other genetic variations may play a role in the pathogenic phenotype. 